Case Report

\title{
General anaesthesia in the dental management of a child with cerebral palsy and autism: A Case Report
}

\author{
Seno Pradopo, Firli Cahaya Khairani, Wahyudi Sudarsono, Masyithah, Udijanto Tedjosasongko \\ Department of Pediatric Dentistry, \\ Faculty of Dental Medicine, Universitas Airlangga, \\ Surabaya - Indonesia
}

\begin{abstract}
Background: Cerebral palsy described as a spectrum of movement and posture disorders which result from pathological injury to the developing fetal or infant brain. Many conditions associated with cerebral palsy require surgery. Purpose: This article presents case report of a 15-year old, autistic male patient with cerebral palsy who required urgent management of multiple gangren radix, multiple pulpitis and gingivitis. Case Management: General anaesthesia was chosen for the purpose of behaviour management. The dental procedure were restoration with flow able composite of 7 teeth, extraction of 15 gangren radix, scaling of maxilla and mandibular region, also preventive treatment with topical aplication of fluoride. Discussion: Principles to the care of a such patient including early recognition, parental support, multi-disciplinary planning of procedure requiring general anesthesia, continuity of anesthesia care, and clear guidelines about the perioperative management of uncooperative children. Dental surgery under general anaesthesia was done, the patient was received restoration with flow able composite of 7 teeth, extraction of 15 gangren radix, scaling calculus at maxilla and mandibular region, also preventive treatment with topical aplication of fluoride. Conclusion: The case report showed that dental treatment should be done in patient with cerebral palsy and autism comprehensively.
\end{abstract}

Keywords: Cerebral Palsy, Autism, General Anaesthesia, child, dental treatment

Correspondence: Seno Pradopo, Departement of Pediatric Dentistry Faculty of Dental Medicine, Universitas Airlangga, Jl. Prof. Dr. Moestopo 47 Surabaya 60132 - Indonesia. Phone: +62315030255 Email: senop@fkg.unair.ac.id

\section{INTRODUCTION}

Special needs are terminology used in clinical diagnostic and functional development to describe individuals who require assistance for disabilities that may be medical, mental, or psychologicall. As medical care providers, we should individualize our treatment and service and ensure delivery of optimal medical resources for individuals with special needs. ${ }^{1}$ There is a higher incidence of dental caries in patients with $\mathrm{CP}$ because of inadequate plaque removal due to motor, sensory, or intellectual disabilities in these patients. Oral health education and the interventions of sedation and general anesthesia should be instituted for management of dental health conditions. ${ }^{1}$

Cerebral palsy (CP) is a collective term used to describe a diverse group of neurological disorders characterized by varying degrees of motor, sensory, and intellectual impairmentt. The National Institute of Neurological Disorders and Stroke published a perinatal study which suggested that complicated deliveries could only account for $10 \%$ of all CP cases. The life expectancy of many patients with $\mathrm{CP}$ now extends into adulthood and they frequently present to both specialized and general hospitals for anesthesia and surgery. ${ }^{2}$

Autism spectrum disorder (ASD) refers to a group of disorders of brain development, which are variably characterized by social interaction and communication difficulty, restricted interests, and repetitive behaviors. Common behavior-related barriers to dental care include the fact that children with ASD are prone to agitation, self-injury, and 


\section{Indonesian Journal of Dental Medicine}

Volume 1 Issue 1 2018; 1-4

emotional dysregulation. Hypersensitivity to sensory input can also interfere with dental and oral care. These features make it difficult for parents to care for their children's teeth and for dental professionals to position, examine, and treat children with ASD. ${ }^{3}$ This article presents case report of a 15-year old, autistic male patient with cerebral palsy who required urgent management of multiple gangren radix, multiple pulpitis and gingivitis.

\section{CASE}

A 15-year old, (weight: $37 \mathrm{~kg}$ ) male patient with cerebral palsy and autism accompanied by his mother and grandmother to the Pediatric Dentistry Clinic, Universitas Airlangga Dental Hospital with a major complaint of recurrent painful and abscessed in mandibula molar permanent. Extra oral examination showed simetris face (Fig.1), intra oral examination showed multiple gangren radix, multiple pulpitis and gingivitis.

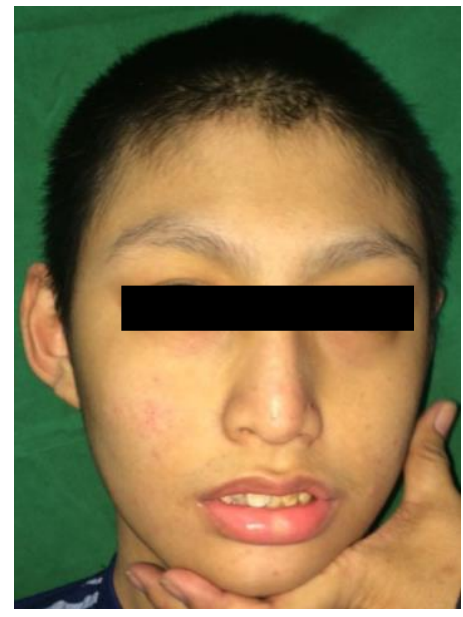

Fig 1. Extra oral photo

Due to his lack of ability to communicate and cooperate with others, Pedi-wrap and oral opening devices were used. Treatment was performed step-by-step while the patient was under physical restraint. During a regular oral examination, a number of dental caries and gangren radix were identified on the maxillary and mandibular tooth (Fig.2), and accordingly, exctraction and restorative treatment was planned.

It was deemed that the caries would develop rapidly due to patient's poor oral hygiene status, and that performing dental procedures on multiple teeth using local anesthesia would be difficult due to the patient's lack of ability to cooperate. As a result, general anesthesia was chosen for the purpose of behavior management. His mother and grandmother were well informed about the method, process, and side effects of the dental procedure. Then, a consent form was signed prior to inpatient general anesthesia procedure.

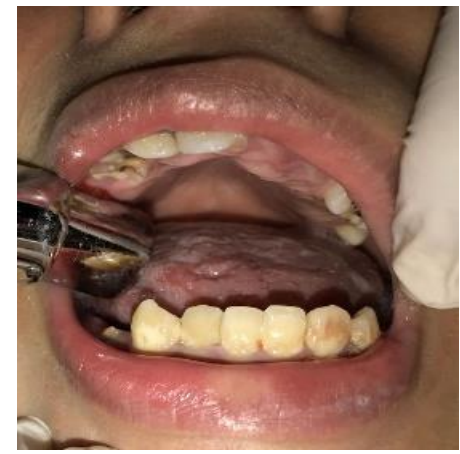

Fig 2. Preoperative intra oral photo

A preoperative complete blood examination was performed and consultation was requested of his private pediatrics. The test results showed no risk factors associated with performing general anesthesia on the patient. At this time, the patient was not taking any medications. Monitoring devices (electrocardiogram [ECG] monitor, pulse oximeter, sphygmomanometer, thermometer, and end-tidal $\mathrm{CO}_{2}$ monitor) were attached to the patient. Antibiotic cefazolin 2 gr as a prophylaxis, drip in NaCL $0.9 \% 100 \mathrm{ml}$ one hour before procedure for 15 minutes. No movement or spontaneous respiration by the patient was observed during the procedure. No additional muscle relaxants were administered. The dental procedure were restoration with flow able composite of 7 teeth, extraction of 15 gangren radix (Fig.3), scaling of maxilla and mandibular region, also preventive treatment with topical aplication of fluoride. Socket were sutured with absorbable string. The treatment was take 2 hours long and the anesthetic procedure lasted for 2,5 hours. The patient showed no signs of complications after the treatment and was moved back to his house, one day after dental surgery. 


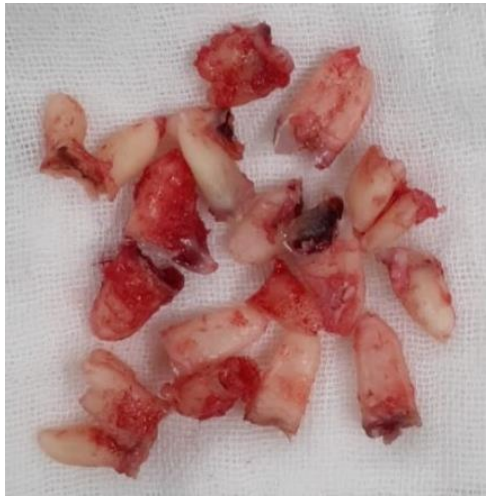

Fig 3. Extracted gangren radix photo

One week follow up showed poor oral hygiene status from the intra oral examination (Fig.4). His mother and grandmother were instructed how to perform dental hygiene procedure by daily cleaning of the teeth with sterile gauze. The patient was scheduled for follow up in the next 3 months.

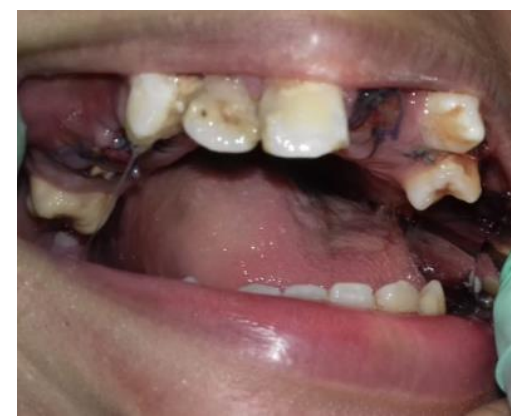

Fig 4. Intra oral photo a week postoperatif

\section{DISCUSSION}

Appropriate general anesthesia could ease the process of dental treatment in patients who are unable to cooperate during the dental visit. Motor dysfunction includes Parkinson, cerebral palsy, and other diseases that cause uncontrolled tremor. General anesthesia is sometimes required so that these patients can keep their mouth open for the treatment.

Cerebral palsy comprises a group of no progressive motor conditions manifested by physical disabilities in development due to brain injuries during the antenatal, perinatal, or postnatal period. The prevalence is approximately two per 1000 live births. Patients with cerebral palsy tend to have complicated dental problems due to lack of lip seal, higher malocclusion rate, temporomandibular disorder, difficulty swallowing, and associated malnutrition and aspiration pneumonia. Their oral hygiene status is also the burden of the caregivers.

Patients with cerebral palsy usually have additional disability attributable to central nervous system damage, such as cognitive impairment, visual or hearing problems, seizures, and communication and behavioral disturbances, as well as the chronic systemic problems resulting from their disease. Severity of cerebral palsy is often associated with postoperative complications. Airway maintenance during anesthetic induction may be complicated by excessive secretions. Tracheal intubation should be performed if this is a concern or if there is a history of gastroesophageal reflux. Tracheal tube size selection should be based on the patients' age. Careful positioning is of paramount importance in the child with spastic cerebral palsy to forestall nerve or muscle damage. Fixed contractures may add difficulty to positioning.

The responses to anesthetic agents may also differ. They may be resistance to nondepolarizing muscle relaxants. They appear to be a close correlation between severity of preoperative cerebral palsy and postoperative complications. The risk of perioperative adverse events was $63.1 \%$, mostly of hypothermia and hypotension. Factors associated with increased risk included American Society of Anesthesiologists (ASA) physical status score of 2 or higher, history of seizures, upper airway hypotonic, general surgery procedures, and adulthood. ${ }^{1}$

Autism is a developmental disorder that is usually diagnosed before age 3 years. Children with autism have characteristic symptoms such as impaired social interactions, verbal and nonverbal communication deficiencies, limited activities and disinterest, repetitive behaviors, and difficult responses to changes in routine. Some of them have other behavioral disturbances such as self-mutilation, aggression, and psychiatric symptoms. Some autistic patients take medications for behavior control to help them integrate effectively in the educational and rehabilitative process. Early communication with the patient's families, flexibility to individualize the anesthetic plan, and awareness of possible interaction of behavior medications and 
anaesthetics are important in management of these patients. ${ }^{1}$

Increasing numbers of dental procedures that involve anesthesia as a means to manage patient behavior are being performed today, and common anesthetics are used in outpatient clinics during general anesthesia due to their rapid onset of action and good reversibility upon ceasing the administration. ${ }^{5}$ In the hospital this study focused on, it was also common for general anesthesia to be performed on intellectually disabled patients, who are unlikely to cooperate during dental procedures in an outpatient operation room. ${ }^{4}$

However, in cases of outpatient general anesthesia, it is difficult to check a patient's comorbidities unlike in inpatient general anesthesia, and the cooperation of the patient's is necessary for the proper preparation of general anesthesia and detection, as well as management, of complications that may occur after the patient is discharged.

It is known that for intellectually disabled children, voluntary oral care is hard to achieve. Therefore, it is important to treat dental problems in intellectually disabled pediatric patients, as well as to teach parents about oral care for children in a way that is easy for them to understand. Through communication with not only patients, but also their parents, an environment in which patients can receive positive oral care over a long-term period should be created. For intellectually disabled patients undergoing dental procedures, it possible variations in the method of general anesthesia should be considered depending on their parents' level of cognitive skills. It is important to talk to patients and their guardians about the treatment and anesthetic procedures, as well as their side effects and risk/benefits to help them reach their own decision.
Informed consent is an important factor of a patient's autonomous decision-making, and it prevents medico legal issues related to various complications. Most children have immature cognitive abilities, and cannot make legal decisions on their own; therefore, informed consent must be given by their legal representatives. To prevent any legal issues from arising later on, several factors should be considered, such as determining the person to give informed consent and the level at which the surgical and anesthetic procedures should be explained to intellectually disabled patients. The case report showed that dental treatment should be done in patient with cerebral palsy and autism comprehensively.

\section{REFERENCES}

1. Wang YC, Lin $\mathrm{IH}$, Huang $\mathrm{CH}$, Fan SZ. Dental anesthesia for patients with special needs. Acta Anaesthesiologica Taiwanica 2012; 50; 122-5.

2. Prosser DP, Sharma N. Cerebral palsy and anaesthesia. Advance Access publication 2010; 72-6.

3. Lewis C, Vigo L, Novak L, Klein EJ. Listening to Parents: A Qualitative Look at the Dental and Oral Care Experiences of Children with Autism Spectrum Disorder. Pediatric Dentistry; 2015; 37; E89-104.

4. Han JH, Hyun HK, Kim YJ, Kim JW, Jang KT, Kim CC, Lee SH, Shin TJ. Dental treatment under general anesthesia in an intellectually disabled child with intellectually disabled parents. J Dent Anesth Pain Med 2016; 16; 213-6.

5. Dougherty N. The dental patient with special needs: a review of indications for treatment under general anesthesia. Spec Care Dentist 2009; 29: 17-20. 